\title{
FRACTIONAL ORDER BUTTERWORTH FILTER FOR FETAL ELECTROCARDIOGRAPHIC SIGNAL FEATURE EXTRACTION
}

\author{
Hadi Mohsen Alkanfery ${ }^{1}$ and Ibrahim Mustafa Mehedi ${ }^{2}$ \\ ${ }^{1}$ Electrical and Biomedical Engineering, \\ King Abdulaziz University, Jeddah, Saudi Arabia \\ ${ }^{1}$ Medical Equipment Specialist, Ministry of Health, Najran, Saudi Arabia \\ ${ }^{2}$ Electrical and Computer Engineering, King Abdulaziz University, \\ Jeddah, Saudi Arabia
}

\begin{abstract}
The non-invasive Fetal Electrocardiogram (FECG) signal has become a significant method for monitoring the fetus's physiological conditions, extracted from the Abdominal Electrocardiogram (AECG) during pregnancy. The current techniques are limited during delivery for detecting and analyzing fECG. The nonintrusive fECG recorded from the mother's abdomen is contaminated by a variety of noise sources, can be a more challenging task for removing the maternal ECG. These contaminated noises have become a major challenge during the extraction of fetal ECG is managed by uni-modal technique. In this research, a new method based on the combination of Wavelet Transform (WT) and Fast Independent Component Analysis (FICA) algorithm approach to extract fECG from AECG recordings of the pregnant woman is proposed. Initially, preprocessing of a signal is done by applying a Fractional Order Butterworth Filter (FBWF). To select the Direct ECG signal which is characterized as a reference signal and the abdominal signal which is characterized as an input signal to the WT, the cross-correlation technique is used to find the signal with greater similarity among the available four abdominal signals. The model performance of the proposed method shows the most frequent similarity of fetal heartbeat rate present in the database can be evaluated through MAE and MAPE is 0.6 and 0.041209 respectively. Thus the proposed methodology of de-noising and separation of fECG signals will act as the predominant one and assist in understanding the nature of the delivery on further analysis.
\end{abstract}

\section{KEYWORDS}

Fetal Electrocardiogram (FECG), Fractional Butterworth Filter (FBW), Fetal Heart Rate (FHR), pregnancy, Abdominal Electrocardiogram (AECG), Non-invasive.

\section{INTRODUCTION}

Among $80 \%$ of perinatal events, $20 \%$ of pregnancies are high risk, whereas the rest are preventable as per the record of the World Health Organization (WHO). Intelligent detection and analysis of fECG signals help in identifying the preventable ones at the earlier stage. Furthermore, fetal deaths worldwide are 2.65 million annually, with $45 \%$ of intra-partum deaths can occur. Heart defects are the primary cause of mortality. Nine out of 1,000 infant children in the UK are estimated to have a congenital cardiopathy at birth [1]. Using Fetal heart rate activity many scientific community has developed a brilliant technologies to control the physiological status of the fetus [2,3]. It is possible to obtain valuable knowledge about the physiological condition of the fetus by analyzing the fECG waveform. The morphological characteristics of fetal ECG can be successfully identified using an invasive approach involving an electrode 
Signal \& Image Processing: An International Journal (SIPIJ) Vol.12, No.5, October 2021

connected to the fetal scalp. The direct fetal ECG measurement is very time dependent method that can only be recorded after the membrane ruptures. There are various electrodes mounted on the mother's abdomen that may also be used to extract fECG from AECG recordings. The accuracy of fetal heart parameters has recently been demonstrated by an ECG record as well as their excellent adherence with the values of scalp electrodes. Electro hysterogram (EHG), Maternal ECG, and fECG are the electrical activity of the uterus that is very strong during childbirth in which electrophysiological signals are presented in each measurement. Since fetal ECG seems to be combined with several other causes of disturbance including maternal muscular noise, skin resistance interference, acoustic noise, respiration caused by fetal movement, and power line distribution. Today, the fetal monitoring is completely based on the FHR monitoring assisting Doppler \& Ultrasound/ Sonography techniques only and does not integrate the characteristics of the fECG waveform. The fECG signal provides useful information to define the variability of FHR and further measures for heart function. Cord compression, fetal heart block, fetal arrhythmia, fetal malposition such as Asphyxia, Bradycardia, congenital heart disease, tachycardia, hypoxia, and some other abnormal situations can also be detected by the fECG analysis [3]. The ranges of FHR should be between 120 and 160 beats per minute. Bradycardia has the FHR as less than 120 beats and Tachycardia has the FHR greater than 180 beats per minute. At last, oxygen levels have been associated with changes in the width of the QRScomplex, PR, and PQ intervals, the P-wave, T-wave, and ST-segment [4].

To utilize the advantages of such a Non-invasive method, several signal processing techniques to retrieve the fECG from the non-invasive recordings are developed. Besides this, fetal electrocardiography has not been demonstrated to be an effective method to imagine such major flaws even during labor. Rather, fetal electrocardiography is often used to diagnose more common problems including general ischemia caused by fetal positioning that suffocates the umbilical cord. The noninvasive FECG is contaminated by movement objects, fetal brain activity, myographic (muscle) signals, and several layers of dielectric physiological environments the electrical signals must pass, which is the reason for this restriction. So, an efficient algorithm for extraction fECG from AECG providing an exact cardiograph (morphology) is required.

This research work introduced the design of FBWF is applied for pre-processing of FECG from the multichannel abdominal ECG recordings in complex w plane. Initially, FBWF has been built in the w-plane rather than the complex s-plane with integer-order filters. Then the definition of fractional order derivatives has been discussed with the stability of w-plane linear fractionalorder systems. Then fast ICA (FICA) algorithm with the combination of fast Fourier Transform (FFT) and the undecimated Wavelet transform (WT) was successfully used to extract FECG from AECG separation with constant coefficients that changes the weights according to the signal. Finally, to measure the FHR, fetal R-waves are observed. The R-R intervals are reliable in duration based on the extracted from the various fECG signals. This study offers a numerical representation of HR variations around a mean, which reflects a subject's average HR. It is shown that the HR variability defines the capacity of the heart to respond to and control various stimuli. This research work aims to monitor the fetus physiological conditions which are non - intrusive fECG recorded from the mother's abdomen during the pregnancy.

The organization of the paper is as follows. Section 2 presents a literature survey based on various fetal ECG extraction methods, Section 3 describes a proposed methodology based on preprocessing, extraction of fetal ECG using FICA, post-processing, and analysis of Fetal ECG feature extraction stage, Section 4 describes experimental results, Section 5 describes performance evaluation and Section 6 ends with a conclusion. 


\section{LiterATURE REVIEW}

In recent studies, many researchers have addressed the techniques based on extraction of Fetal ECG during pregnancy. Initially, this section focuses survey based on extracting fECG such as the Adaptive filtering technique, WT, and features for analyzing fetal heart rate. Since an examination of the fECG, which allows for high efficiency and precision in measuring FHR, there has been an increasing interest in tracking fetal cardiac bioelectric activity for many years. Invasively recording the fECG are directly from the fetal head while labor whereas noninvasively recording the fECG are indirectly mounted on the maternal abdominal wall from electrodes during labor [5]. The amplitude of the Fetal QRS complex (FQRS) in abdominal signals exceeds $20 \mathrm{~V}$ and is highly influenced by the maternal BMI. There are various muscle interference are available but the most strong and efficient is Maternal Electrocardiogram (MECG) with a significantly larger amplitude [6]. The fECG signal remains undesirable with further study due to incomplete MECG suppression [7], FHR determination, preventing the identification of FQRS complexes, and accurate location of R waves. During labor, an abdominal fECG and a reference direct fECG had been recorded at the same time. The clinical experts corrected reference pregnancy signal data obtained from an automated detector. The resulting data set contains a wide range of clinically relevant FHR interferences, which can establish new techniques for the study of fECG signals and eventually promote the use and accuracy of abdominal ECG [8]. To extract FECG signal from AECG signal by utilizing a FIR Adaptive Noise Canceller (ANC) with adaptive algorithms to update the filter coefficients. Adaptive filters are suitable for the current problem of interest and Least Mean Square (LMS) are analyzed for the problem of fECG extraction [9]. The benefit of using FIR ANC is that it has a lower computational cost and performs better with two input signals, one of which is primary and the other is reference, as opposed to other non-adaptive techniques such as PCA and ICA, which can only provide better performance when multichannel input is present. To update adaptive filters in ANC, LMS and RLS algorithms can be employed. In the literature, both LMS and RLS algorithms for FECG extraction may be discovered. [10]. To handle this challenge, several adaptive filtering and artificial intelligence algorithms are used. As an intelligent system, complex real-world problems require a combination of information, skills, and approaches from diverse sources. That intelligent system should have human-like abilities, be able to adapt to changing environments, and learn to improve on its own. [11]. Using an ANC device, the extracted signal of the FHR from the mother was enhanced. It employs widely used adaptive Normalized LMS algorithms (NLMS). Fetal extraction and de-noising are suggested in this paper. The NLMS algorithm is combined with the Savitzky-Golay (SG) filter in this system [12]. Wavelet decomposition and an ICA algorithm are used to develop a novel approach. The proposed method was evaluated and attain $96 \%$ accuracy in detecting the R wave of fECG, which is capable of future research [13]. To remove fetal ECG from the mixed-signal, Vaidya and Chaitra describe comparing the three separate adaptive filters namely Kalman filter, LMS, and NLMS. A required inference is reached by comparing the characteristics such as computational complexity, reliability, signal-to-noise ratio, and stability [14]. MATLAB was used to apply fetal extraction procedures and the fetal ECG was successfully extracted. The Fast fixed-point approach for Independent Component Analysis (FastICA) has been widely employed in fECG extraction. The FastICA algorithm affects the convergence which is responsive to the initial weight vector. To address this problem, an improved Fast ICA method for extracting fetal ECG was proposed [15]. Adaptive filtering [5], [16], wavelet analysis [17], matched filtering [18], blind source separation [19], ICA [20], neural network [21], [22], and Singular Value Decomposition (SVD) [23] are the most common fetal ECG extraction algorithms are currently accessible. In case that the source signals are statistically independent, ICA can isolate the source signals from the mixed signals, without the need for information concerning the source signals and the combined matrix. ICA is thus regarded as an advantageous tool for fetal ECG extraction. 
Signal \& Image Processing: An International Journal (SIPIJ) Vol.12, No.5, October 2021

Researchers have suggested several improving ICAs in recent years, allowing non-Gaussian signals to be separated.

\section{Materials AND Methods}

This research work consists of three major stages. Initially, preprocessing of the signal is done by using FBWF which is mainly used to eliminate interfering signals such as certain physiological signals, baseline wander, and power line signals. Then cross-correlation can be used to select the reference signal as a Direct ECG and the abdominal signal as an input signal based on the greater similarity between them. Then the extracted signals from Direct and abdominal ECG can be processed by wavelet transform. This wavelet and the FICA decomposition property helps to eliminate noise from abdominal signals as well as separate fetal ECGs from the maternal ECG. After, the extracted features such as QRS peaks were analyzed for FECG signal which is performed to validate with sample heart rate from the database. The general block diagram of the work presented in this paper is shown in Figure.1.

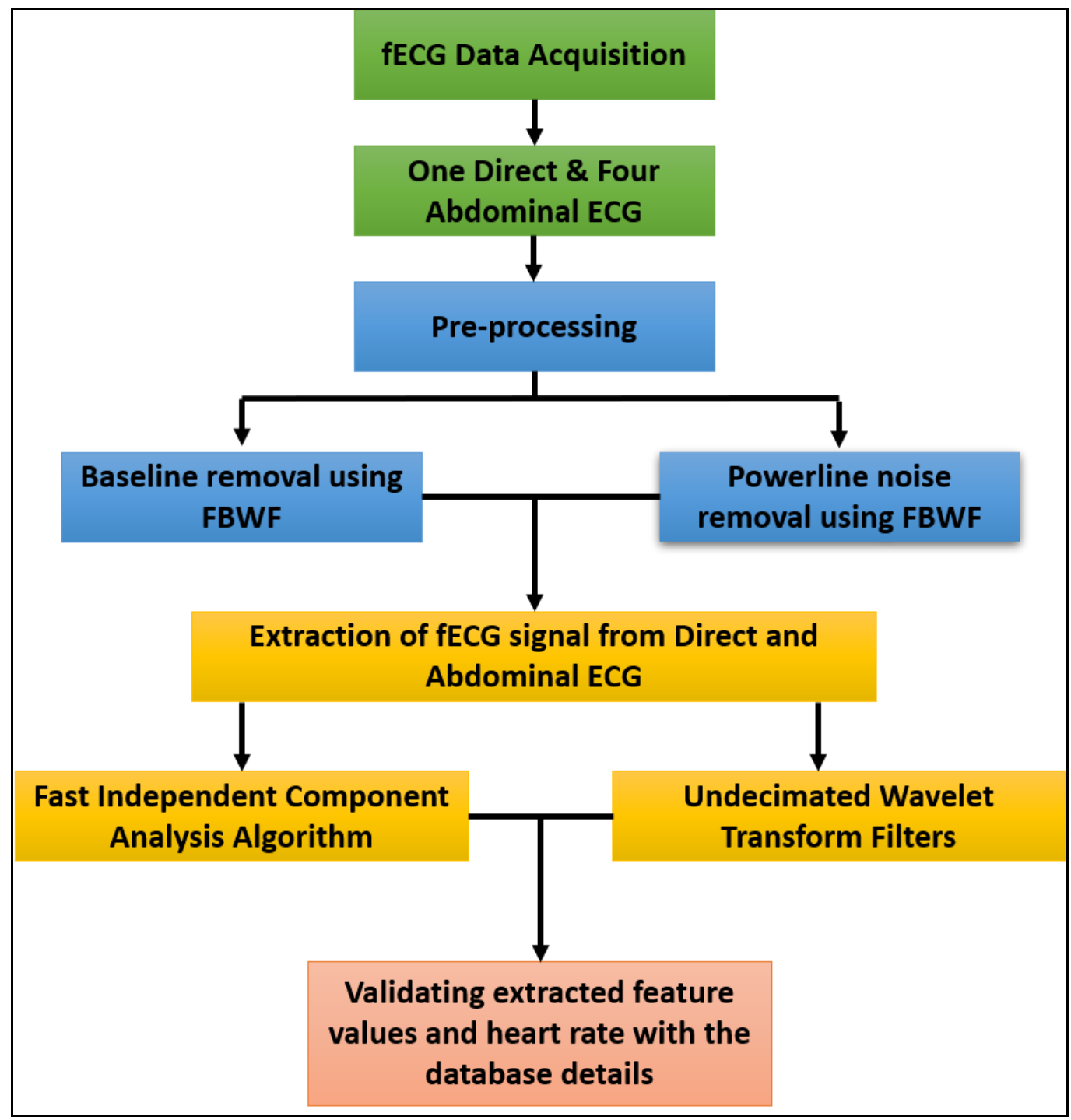

Figure 1. Flowchart of the research work 
Signal \& Image Processing: An International Journal (SIPIJ) Vol.12, No.5, October 2021

\subsection{Pre-processing}

The noise from the input fetal ECG signal is removed during preprocessing by applying fractional-order Butterworth Filter with the suitable and precise cut of frequencies. The signal-tonoise ratio is minimal during pregnant women since the FHR is just a few $\mathrm{uV}$ in amplitude whereas interfering signals may be represented as hundreds of $\mathrm{uV}$. As a result, FBWF was built in the w-plane to remove interfering signals. In this paper formulated $\mathrm{W}$ plane as ( $\mathrm{w} 1 / 4 \mathrm{sq} ; \mathrm{q} \in \mathbb{R}+$ ) which contains all the possible types of poles such as under, hyper and ultra-damped were also taken into consideration. In the w-plane, diagnostic design of the filter has been performed, as well as certain criteria for the position of the poles such as stable and unstable have been determined. In the case of fractional order, derivation found the principle of classical Butterworth filter which are kept unchanged whose radius is equal to cut off frequency whereas the poles are located along with the perimeter of the circle. As a result, the suggested formulation takes into account all of the stable poles. The relation $\mathrm{s}=$ can be used for the w-plane by obtaining the transfer function and then mapped back into the s-plane. To confirm the maximally flat Butterworth existence, the corresponding reaction curves are obtained. In the fractional domain, the resulting complexity would make more difficult generalization filters and fabrication costs are outweighed by the benefits of greater flexibility. Hence the designing system has been adopted by considering a relative tradeoff between accuracy and complexity. Therefore, the filter has truncating up to the first decimal place which is better to realize whose overall order is a fraction. To improve the characteristics of the frequency domain the accuracy can be increased by intuitive judgments. The FBW filter considers the stability of fractional linear systems which has been developed on the line of integer order BW filter [24].

The goal of this study is to extract clean fetal electrocardiogram (fECG) signals from noninvasive abdominal ECG recordings for monitoring the health of the fetus during pregnancy and childbirth. The proposed extraction was implemented in MATLAB ${ }^{\circledR}$ by processing the abdominal and direct ECG with a fractional Butterworth filter for the separation of direct fetal ECG signals.

\subsection{Data Collection}

The data obtained from the Abdominal and Direct Fetal Electrocardiogram Database on PhysioNet is used in this work [23]. Referring to patient 1 and patient 2, a set of multichannel electrocardiogram (ECG) recordings acquired from two distinct women in labor between 38 and 41 weeks of pregnancy, were used in this study. By using the KOMPOREL system for fetal ECG manipulation and processing, those recordings were obtained in the Department of Obstetrics at the Medical University of Silesia (ITAM Institute, Zabrze, Poland) [13]. Four distinct signals were obtained from the maternal abdomen for each recording, as well as from the fetal head a reference direct fetal ECG is recorded. All recordings were selected at $1 \mathrm{kHz}$ and coded in 16 bits, with a total duration of 5 minutes. Figure 2 depicts the Investigation of moral characteristics and also the extraction of the fetal electrocardiogram as the technical outline format. The signal recorded from the mother's abdomen and from the fetal head are pre-processed using FBWF. The pre-processed signal is then used to extract the fetal ECG with the help of FICA and Wavelet transform technique. 


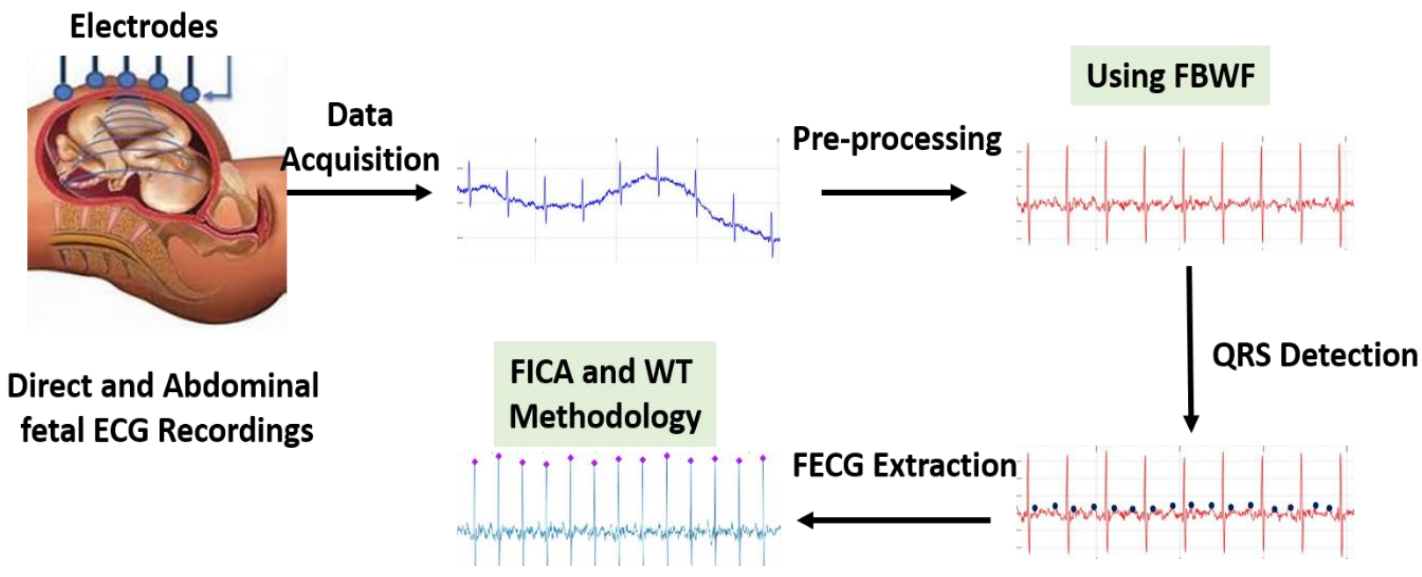

Figure 2. System diagram of FECG extraction

\subsection{Phases of pre-processing}

Typically, the range of the EHG signal spectrum is between $0.1 \mathrm{~Hz}$ to $3 \mathrm{~Hz}$ in each signal [14]. We used the MATLAB signal processing toolbox to create a high-pass Fractional Butterworth Filter (FBWF) with a stopband edge frequency of $0.5 \mathrm{~Hz}$ and a passband edge frequency of $3 \mathrm{~Hz}$ to eliminate baseline wandering and cancel most EHGs. A narrow band noise of less than $1 \mathrm{~Hz}$ centered at $50 \mathrm{~Hz}$ is the interference of the power line. We utilized a severe notch filter (Q-factor equal to 30) at $50 \mathrm{~Hz}$ to minimize this noise kind. Furthermore, we constructed a 100th order IIR Butterworth filter from our bandwidth range $(0.5-34) \mathrm{Hz}$ to reduce high-frequency noises from motion artifacts, as the frequencies of relevance for PQRST wave mining are predominantly situated in the domain.

\subsection{Methods of Independent Component Analysis}

To breakdown the multivariable signal into a set of mutually separate non-Gaussian elements, a statistical method of Independent Component Analysis (ICA) is used, provided that the observed signals are mathematically specified by the ICA model [5], as a mixture of independent sources; $\mathrm{x}=\mathrm{A} \cdot \mathrm{s}$, where $\mathrm{x}=\left[\mathrm{x}_{1}, \mathrm{x}_{2}, \mathrm{X}_{3}, \ldots \ldots \mathrm{x}_{\mathrm{m}}\right]^{\mathrm{T}}$ is the noticed multivariate signal, $\mathrm{s}=\left[\mathrm{s}_{1}, \mathrm{~s}_{2}, \mathrm{~s}_{3}, \ldots \ldots \mathrm{s}_{\mathrm{n}}\right]^{\mathrm{T}}$ is the original unknown multivariate source signal, $\mathrm{M}$ is the number of noticed signals, $\mathrm{N}$ is the number of sources and $\mathrm{A}$ is the mixing matrix. In order to acquire estimated independent components $y$, the goal of ICA is to return the linear unmixing matrix $W$ is such that: $y=W . x$ Neural algorithms have many benefits towards the Fast ICA they are; It is parallel, Evenly, distributed, Simple in computation and consumes less memory space. Moreover, independent components, approximately equal to projection, may be estimated one by one [5]. The TSA (Time Sample Analysis) independent component analysis VI with which fast ICA approach is readily adopted thanks to its convergence speed, as offered by the advanced signals processing toolbox.

\subsection{Post-processing}

The wavelet function in 5 levels of decomposition enhanced the Fast ICA extraction quality for ECG signals [15], using biorthogonal 4.4. The denoise stage of wavelets transformed. We have used Undecimated Wavelet Transformation (UWT) from the Wavelet Denoise toolbox to remove the multiband noise from ICA extracted fECG signals. A biorthogonal wavelet filter was chosen at eight decomposition levels to better identify the acquired signal, and its scaling function is intricately linked to the shape of the ECG. 
Signal \& Image Processing: An International Journal (SIPIJ) Vol.12, No.5, October 2021

Regrettably, even after effectively de-noising with wavelet transforms, some noise components remained, necessitating the use of additional filtering techniques must be needed to increase the signal-to-noise (SNR) value. We used FFT and Inverse FFT from the Signal Processing toolbox to create a signal processing pair consisting of Fast Fourier Transform (FFT) and Inverse Fast Fourier Transform (IFFT). Signals were translated from time to frequency domain using the FFT transform, with high and low-frequency components sorted out to highlight undesired noise components. In the frequency domain, the signal is separated from the first and final $5^{*} \mathrm{FFT}$ length/sampling rate samples and therefore filtered. After that, the filtered signal is converted back to the time domain and ready for feature extraction by using IFFT.

\subsection{ECG of a fetus with extraction stage}

We used a combination of Peak Detector from Signal Operation toolbox and WA Multiscale Peak Detection from Wavelet Analysis toolbox to extract fECG features to detect QRS peak positions and maximum amplitudes. Using the Extract Heart Rate, FHR may be detected automatically for various time intervals of recordings.

\section{RESUlt AND DisCuSSION}

MATLAB ${ }^{\circledR}$ tool is used for extracting fECG for analyzing fetus heart rate. For a better analysis of the algorithm, the ECG acquired in a real-time scenario will be the best choice. Physionet provides free web access to PhysioBank, which contains a large collection of recorded physiologic signals.

The recordings in EDF format have already been preprocessed by filtering for noise removal and an excellent source of data for evaluating new fECG processing techniques. The database used for Non-invasive fetal electrocardiogram is involved with the data of 55 series in multichannel abdominal recordings that have been collected from 10 subjects among the pregnancy period of 21 to 40 weeks. The implemented algorithm is tested on 10 records having a better trace. Figure. 3 (A) and (B) have discussed Direct and followed by abdominal 1, 2, 3, and 4 signals. Figure. 3 (A) corresponding to 1 clinical case of raw ECG signal which contains few $\mathrm{uV}$ in amplitude whereas interfering signals may be represented as hundreds of $u V$. Figure. 3 (B) shown maternal ECG signal after removal of hundreds of $\mathrm{uV}$.
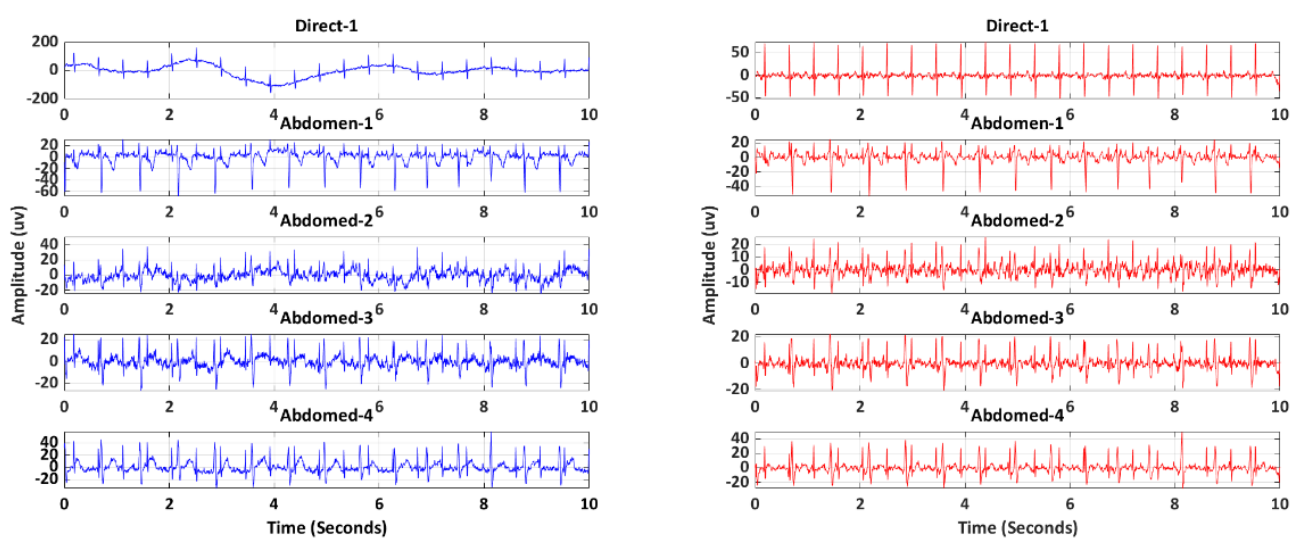

Figure.3. (A) Raw ECG signal (B) Pre-processed ECG signal

Figure 4 shown post-processing using FICA and undecimated WT which contains all the QRS complexes are extracted. The resulting signal obtained fetal ECG which can be separated from 
Signal \& Image Processing: An International Journal (SIPIJ) Vol.12, No.5, October 2021

the mother's ECG. The red color indicates fetal ECG whereas the blue color indicates mother ECG.

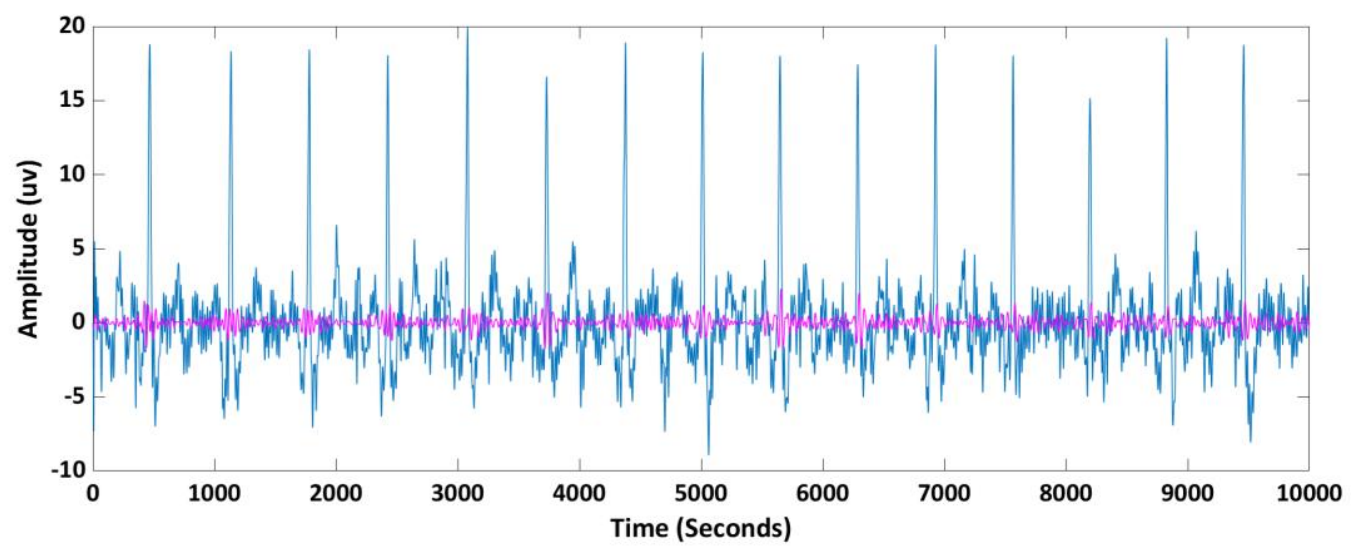

Figure 4. The resulting signal of Fetal ECG separation

\subsection{Performance Evaluation}

In Figure 5, the analysis of the heart rate signal extracted from fECG is shown. It also recognizes time slots that increase or decrease in accordance with fetal heart rate normal ranges, with $\mathrm{R}$ waves indicated by a pink peak.

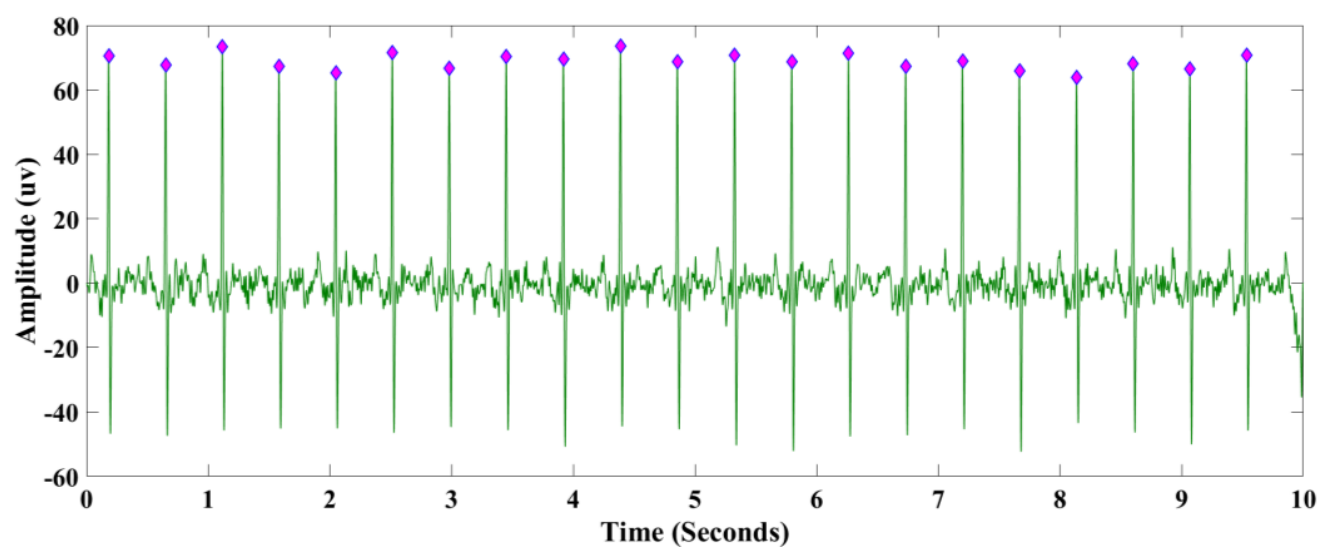

Figure 5. $\mathrm{R}$ waves detection in fECG signal 


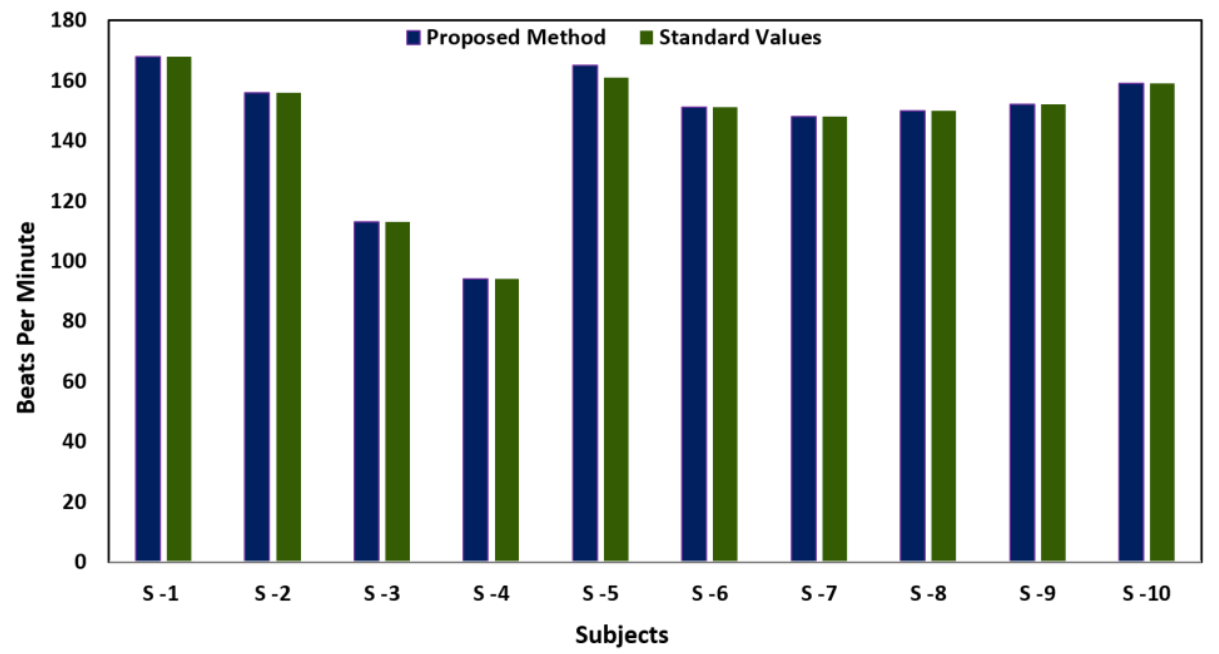

Figure 6. Comparison of beats per minute for 10 different subjects

In figure 6 shows that the proposed method has most frequent similarity except subject 5 which are mismatched in database samples.

\subsection{Calculation of Error Rate}

The average of absolute percentage error was calculated for 10 different subjects using the following formula.

Mean Absolute Error (MAE): The MAE was calculated as the average absolute difference between the fetal heart rate variability of bits per minute for proposed method(Y) and database samples $(\hat{Y})$.

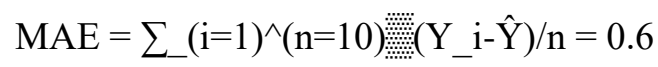

Mean Absolute Percentage Error (MAPE): The MAPE was calculated by averaging the individual absolute percent errors.

$$
\operatorname{MAPE}=\left(\sum \_(\mathrm{i}=1)^{\wedge}(\mathrm{n}=10)\left(\mathrm{Y} \_\mathrm{i}-\hat{\mathrm{Y}}\right) / \mathrm{n}\right) / \mathrm{n} \times 100=0.041209
$$

Therefore the performance of the proposed method can be obtained as 0.6 MAE and 0.0412 MAPE percentage error.

\section{Conclusions}

During pregnancy, one of the best methods of diagnosis is fetus ECG signals which assist in analyzing the fetus status in the uterus. However, the requirement of an automated method to extract the fECG and even analyze by noninvasive recording obtained from the chest and abdomen of pregnant women. Hence, the proposed method is developed based on FICA and the undecimated WT that is majorly focused on extracting fECG signals. These techniques were 
Signal \& Image Processing: An International Journal (SIPIJ) Vol.12, No.5, October 2021

employed for the cancellation of the mother's ECG in such records. The combination of statistical properties and PCA gives a powerful method for studying FECG signals to validate the heart bit rate. The model performance of the proposed method shows the most frequent similarity of fetal heartbeat rate present in the database can be evaluated through MAE and MAPE is 0.6 and 0.041209 . These techniques can be utilized to develop a real-time fECG monitoring and analysis system that helps obstetricians detect fetal distress in pregnant women's wombs and take appropriate measures to help them. In the future, this paper involves qualitative and quantitative analysis based on the threshold value will find the scoring scheme to indicate severe cases of abnormality. Moreover, the extension of this paper utilizes the extracting features of fetal heart rate based on the scoring scheme as the obtained values cross the threshold which depicts highrisk of pregnancy Whereas if the obtained value is equal or less than the threshold depicts a possible risk that is indicated of normal pregnancy.

\section{ACKNOWLEDGEMENTS}

The authors would like to thank everyone who supported this research.

\section{REFERENCES}

[1] J. Behar, F. Andreotti, S. Zaunseder, J. Oster, and G. D. Clifford, "A practical guide to non-invasive foetal electrocardiogram extraction and analysis," Physiological measurement, vol. 37, no. 5, p. R1, 2016.

[2] Mohamed Yacin S and Vennila M, Extended Kalman filtering for fetal ECG extraction using HilbertHuang transform, Int. J. of Biomedical Engineering and Technology, Vol. 18, No. 1, pp 14-29, 2015

[3] G. D. Clifford, I. Silva, J. Behar, and G. B. Moody, "Non-invasive fetal ECG analysis," Physiological measurement, vol. 35, no. 8, p. 1521, 2014.

[4] G. Lamesgin, Y. Kassaw, and D. Assefa, "Extraction of fetal ECG from abdominal ECG and heart rate variability analysis," in Afro-European Conference for Industrial Advancement, 2015, pp. 65-76.

[5] R. Martinek et al., "Non-invasive fetal monitoring: A maternal surface ECG electrode placementbased novel approach for optimization of adaptive filter control parameters using the LMS and RLS algorithms," Sensors, vol. 17, no. 5, p. 1154, 2017.

[6] E. Fotiadou, J. O. Van Laar, S. G. Oei, and R. Vullings, "Enhancement of low-quality fetal electrocardiogram based on time-sequenced adaptive filtering," Medical \& biological engineering \& computing, vol. 56, no. 12, pp. 2313-2323, 2018.

[7] I. Mousavian, M. B. Shamsollahi, and E. Fatemizadeh, "Noninvasive fetal ECG extraction using doubly constrained block-term decomposition," Math. Biosci. Eng, vol. 17, no. 1, pp. 144-159, 2019.

[8] A. Matonia et al., "Fetal electrocardiograms, direct and abdominal with reference heartbeat annotations," Scientific data, vol. 7, no. 1, pp. 1-14, 2020.

[9] C. N. Praneeth, J. D. K. Abel, D. Samiappan, R. Kumar, S. P. Kumar, and P. V. Nitin, "A COMPARISON ON VARIANTS OF LMS USED IN FIR ADAPTIVE NOISE CANCELLERS FOR FETAL ECG EXTRACTION," Biomedical Engineering: Applications, Basis and Communications, vol. 32, no. 04, p. 2050026, 2020.

[10] P. Sutha and V. E. Jayanthi, "Fetal electrocardiogram extraction and analysis using adaptive noise cancellation and wavelet transformation techniques," Journal of medical systems, vol. 42, no. 1, pp. $1-18,2018$.

[11] M. Pradeepa and S. Kumaraperumal, "ADAPTIVE FILTERING AND ARTIFICIAL INTELLIGENCE METHODS ON FETAL ECG EXTRACTION,” Journal of Critical Reviews, vol. 7, no. 7, pp. 295-299, 2020.

[12] A. Ghasemi, F. Shama, G. Karimi, and F. Khosravi, "Recovery and noise cancelling of the fetal heart signal using a combination of NLMS algorithm and SG filter," Circuit World, 2020.

[13] S. L. Lima-Herrera, C. Alvarado-Serrano, and P. R. Hernández-Rodríguez, "Fetal ECG extraction based on adaptive filters and Wavelet Transform: Validation and application in fetal heart rate variability analysis," in 2016 13th International Conference on Electrical Engineering, Computing Science and Automatic Control (CCE), 2016, pp. 1-6. 
Signal \& Image Processing: An International Journal (SIPIJ) Vol.12, No.5, October 2021

[14] R. R. Vaidya and N. Chaitra, "Comparison of Adaptive filters in extraction of Fetal ECG," in 2020 International Conference on Smart Electronics and Communication (ICOSEC), 2020, pp. 10661070.

[15] L. Yuan, Z. Zhou, Y. Yuan, and S. Wu, "An improved FastICA method for fetal ECG extraction," Computational and mathematical methods in medicine, vol. 2018, 2018.

[16] J. Behar, A. Johnson, G. D. Clifford, and J. Oster, "A comparison of single channel fetal ECG extraction methods," Annals of biomedical engineering, vol. 42, no. 6, pp. 1340-1353, 2014.

[17] S. Wu, Y. Shen, Z. Zhou, L. Lin, Y. Zeng, and X. Gao, "Research of fetal ECG extraction using wavelet analysis and adaptive filtering," Computers in biology and medicine, vol. 43, no. 10, pp. 1622-1627, 2013.

[18] A. Dessì, D. Pani, and L. Raffo, "An advanced algorithm for fetal heart rate estimation from noninvasive low electrode density recordings," Physiological measurement, vol. 35, no. 8, p. 1621, 2014.

[19] A. Ghazdali, A. Hakim, A. Laghrib, N. Mamouni, and S. Raghay, "A new method for the extraction of fetal ECG from the dependent abdominal signals using blind source separation and adaptive noise cancellation techniques," Theoretical Biology and Medical Modelling, vol. 12, no. 1, pp. 1-20, 2015.

[20] G. Da Poian, R. Bernardini, and R. Rinaldo, "Separation and analysis of fetal-ECG signals from compressed sensed abdominal ECG recordings," IEEE Transactions on Biomedical Engineering, vol. 63, no. 6, pp. 1269-1279, 2015.

[21] M. Lukoševičius and V. Marozas, "Noninvasive fetal QRS detection using an echo state network and dynamic programming," Physiological measurement, vol. 35, no. 8, p. 1685, 2014.

[22] N. A. Sait, M. Thangarajan, and U. Snehalatha, "Neural network based on Verilog HDL for fetal ECG extraction," International Journal of Biomedical Research, vol. 7, no. 10, pp. 698-701, 2016.

[23] N. Zhang et al., "A novel technique for fetal ECG extraction using single-channel abdominal recording," Sensors, vol. 17, no. 3, p. 457, 2017.

[24] A. Acharya, S. Das, I. Pan, and S. Das, "Extending the concept of analog Butterworth filter for fractional order systems," Signal processing, vol. 94, pp. 409-420, 2014. 


\section{AuTHORS}

Hadi Mohsen Alkanfery received bachelor degree in Biomedical Equipment Technology from Majmaah University, Saudi Arabia. Currently he is pursuing Master of Science in Electrical and Biomedical Engineering in King Abdulaziz University, Saudi Arabia. He also works as a Medical Equipment Specialist in Ministry of Health, Najran, Saudi Arabia.

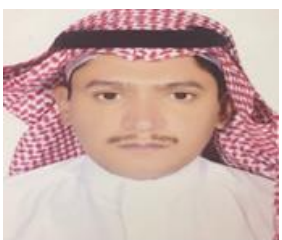

Ibrahim Mustafa Mehedi received B.Sc. in Electrical and Electronic Engineering in 2000 from RUET, Bangladesh. He received the MSc. in Aerospace Engineering from UPM, Malaysia in 2005. Obtaining a Japanese Govt. MEXT scholarship he completed his PhD in Electrical Engineering and Information Systems in 2011 while he was a Research Assistant of the Global Center of Excellence(GCOE) of the University of Tokyo and Japan Aerospace Exploration Agency(JAXA), Sagamihara, Japan. He joined King Abdulaziz University, Jeddah, Saudi Arabia, in November 2012, where he is currently an Associate Professor, and is a research member of the Center of Excellence in Intelligent Engineering Systems (CEIES). Prior to that he worked at KFUPM, and the CocaCola Bottling Plant. His research interests include space robotics, modern control system design and implementation, renewable energy, organic solar cells etc. 